\title{
Management of traumatic brain injury (TBI): a clinical neuroscience-led pathway for the NHS
}

\author{
Authors: Lucia M Li, ${ }^{\mathrm{A}}$ Michael D Dilley, ${ }^{\mathrm{B}}$ Alan Carson, ${ }^{\mathrm{C}}$ Jaq Twelftree, ${ }^{\mathrm{D}}$ Peter ] Hutchinson, ${ }^{\mathrm{E}}$ Antonio Belli, ${ }^{\mathrm{F}}$ \\ Shai Betteridge, ${ }^{G}$ Paul N Cooper, ${ }^{H}$ Colette M Griffin, ${ }^{\text {I }}$ Peter O Jenkins, ${ }^{J}$ Clarence Liu, ${ }^{\text {K }}$ David J Sharp, ${ }^{\mathrm{C}}$ \\ Richard Sylvester, ${ }^{\mathrm{M}}$ Mark H Wilson, ${ }^{\mathrm{N}}$ Martha S Turner ${ }^{\mathrm{O}}$ and Richard Greenwood ${ }^{\mathrm{P}}$
}

Following hyperacute management after traumatic brain injury (TBI), most patients receive treatment which is inadequate or inappropriate, and delayed. This results in suboptimal rehabilitation outcome and avoidable detrimental chronic effects on patients' recovery. This worsens long-term disability, and magnifies costs to the individual and society. We believe that accurate diagnosis (at the level of pathology, impairment and function) of the causes of disability is a prerequisite for

Authors: ANIHR clinical lecturer, Imperial College London, London, UK and UK DRI Care Research \& Technology Centre, London, UK; ${ }^{B}$ consultant neuropsychiatrist, Atkinson Morley Regional Neuroscience Centre, London, UK and Royal College of Psychiatrists, London, UK; C Consultant neuropsychiatrist and honorary professor, Centre for Clinical Brain Sciences, Edinburgh, UK; ' AHP consultant in neuro-rehabilitation, Homerton University Hospital NHS Foundation Trust, London, UK; ${ }^{\text {E }}$ professor of neurosurgery, University of Cambridge, Cambridge, UK and Royal College of Surgeons, London, UK; F professor of trauma neurosurgery, National Institute for Health Research Surgical Reconstruction Research Centre, Birmingham, UK and Institute of Inflammation and Ageing, Birmingham, UK; ${ }^{G}$ consultant clinical neuropsychologist, St George's University Hospitals NHS Foundation Trust, London, UK; ${ }^{H}$ consultant neurologist, Manchester Centre for Clinical Neurosciences, Manchester, UK; ' 'consultant neurologist, St George's University Hospitals NHS Foundation Trust, London, UK; ' consultant neurologist, Epsom and St Helier University Hospitals NHS Trust, London, UK, St George's University Hospitals NHS Foundation Trust, London, UK and Imperial College London, London, UK; ${ }^{K}$ consultant neurologist, Homerton Hospital, London, UK and Barts Health NHS Trust, London, UK; ${ }^{L} \mathrm{NIHR}$ professor and consultant neurologist, Imperial College London, London, UK and UK DRI Care Research

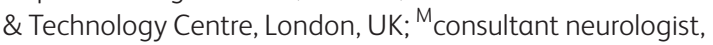
National Hospital for Neurology and Neurosurgery, London, UK;

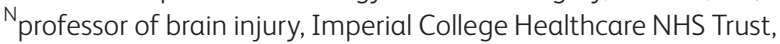
London, UK and Imperial College London, London, UK; ${ }^{\circ}$ principal clinical psychologist and neuropsychologist, Homerton University Hospital NHS Foundation Trust, London, UK; P neurology consultant, National Hospital for Neurology and Neurosurgery, London, UK and Homerton University Hospital NHS Foundation Trust, London, UK appropriate care and for accessing effective rehabilitation. An expert-led, integrated care pathway is needed to deliver accurate and timely diagnosis and optimal treatment at all stages during a TBI patient's care.

We propose the introduction of a specialist interdisciplinary traumatic brain injury team, led by a neurosciences-trained brain injury consultant. This team would engage acutely and for a longer term after TBI to provide accurate diagnoses, which guides subsequent management and rehabilitation. This approach would also encourage more efficient collaboration between research and the clinic. We propose that the current major trauma network is leveraged to introduce and evaluate this proposal. Improvements to patient outcomes through this approach would lead to reduced personal, societal and economic impact of TBI.

KEYWORDS: traumatic brain injury, rehabilitation, neuroscience, pathway, neurology

\section{DOI: $10.7861 /$ clinmed.2020-0336}

\section{Introduction}

Following hyperacute management of traumatic brain injury (TBI), most patients in England receive treatment which is inadequate, inappropriate and delayed. This failure results in increased mortality and worsened long-term disability. These avoidable long-term effects of the injury on patients' physical and mental health lead to significant personal and societal costs. We need a system that allows accurate and timely diagnosis of the complex problems occurring after TBI, enabling rapid and correct treatment, and making it possible to increase and translate research advances into clinical practice.

\section{Purpose}

We describe a new model of care after TBI that is:

$>$ accessed in the acute setting

$>$ focused on pathology and impairment-based diagnostics and therapeutics, as well as function, dependency or cost

$>$ continues in the long term.

This approach, translated from clinical neuroscience, is key to the identification and proper treatment of the complex 
consequences of TBI. It enables a specialist traumatic brain injury consultant (TBIC) and an interdisciplinary traumatic brain injury team (TBIT) to engage acutely after TBI. This team provides diagnoses-informed care, implements appropriate long-term management via a multidisciplinary 'rehabilitation prescription' and encourages the translation of research advances into clinical practice throughout the care pathway. Pilot work carried out in St George's Hospital, Tooting, has found that implementation of a neurosciences-led acute TBI pathway reduces inpatient stay duration and costs for TBI patients (Griffin C, Dilley M, personal communication, 2018). The overall aims of this model are to improve patient outcomes, reduce societal impact of TBI and generate cost savings.

\section{Background}

\section{The scale of the problem}

TBI is a vast and growing health problem for the UK. In England, TBI is responsible for around 900,000 emergency department (ED) attendances and over 200,000 hospital admissions annually. ${ }^{1}$ The rate of 'severe' head injury is $10-20 \%$, with the rest being classified as being 'mild' with no need for surgical or critical intervention or being discharged directly from ED. ${ }^{2}$ Nevertheless, TBI is the single biggest cause of death and disability for those aged $<40$ years, and it particularly clusters in socioeconomically deprived populations, as well as patients with pre-existing health problems, frequent falls and risk-taking behaviour. ${ }^{3,4}$ There are enormous costs associated with TBI, with a total estimated annual cost in Europe of $€ 33$ billion. ${ }^{5}$

TBI pathology is very heterogeneous, and can interact with pre-existing conditions, leading to a wide spectrum of possible problems. For example, an elderly person sustaining an acute-onchronic subdural haematoma occurring after a fall may experience focal weakness arising from the local cortical compression. This may combine, for example, with an underlying peripheral neuropathy secondary to diabetes to create poor mobility. Confusion and poor short-term memory may arise either from injury-associated delirium or from an undiagnosed pre-existing cognitive impairment. On the other hand, a young person whose brain has sustained the shearing forces of a rapid deceleration in a road traffic collision is less likely to experience focal neurological deficits. Rather, their post-traumatic cognitive problems are more likely to be related to the abnormal function of brain networks secondary to the diffuse axonal injury. ${ }^{6}$ In the acute setting, confusion in TBI patients may be secondary to this network damage or post-traumatic amnesia, or even represent metabolic problems (such as alcohol excess). In the chronic setting, poor sleep may be injury-related, or reflect depression or post-traumatic stress disorder. Accurate diagnosis and appropriate management of the many different causes of post-TBI disability require a specialist multidisciplinary team.

Survivors of TBI, particularly after more severe injury, frequently face multiple physical, cognitive, emotional and neurobehavioural problems, which can have long-term and far-reaching consequences (Fig 1). ${ }^{7}$ These include an increased all-cause mortality, loss of independence and employment, relationship breakdown, social isolation, mental illness, addiction and homelessness. ${ }^{4,8}$ There is a high prevalence of previous brain injury within the criminal justice system of between $50-60 \%$ among young offenders. ${ }^{9}$ Neurobehavioural problems, in particular, contribute to poor functional outcomes, high burden on carers and families and predict ongoing long-term healthcare utilisation. 4,10,11 Within the 'mild' TBI population, contrary to popular belief, up to a third (resulting in around 300,000 patients annually in England) can be left with a range of persistent symptoms that prevent them from working and living normally. ${ }^{12-14}$

\section{Current service provision and patient flow}

After trauma, the priority in the UK has been to reduce mortality after moderate/severe TBI, with the focus being on improvement of pre-hospital, acute neurosurgical and critical care, and acute or early inpatient rehabilitation. ${ }^{1,15}$ Since the introduction of the major trauma centre (MTC) system (which seeks to enable early rapid resuscitation and targeted interventions that maximise survival), patient flow and mortality have improved, particularly for those most severely injured. ${ }^{16,17}$

However, it is not enough to save lives; we must also seek to restore them. Hyperacute critical care and surgical treatment must be accompanied and followed by specialist care and rehabilitation to achieve good functional outcomes. Although the major trauma network has led to clear pathways for acute early specialist inpatient rehabilitation ('level 1'; see Box 1), the rehabilitation prescription is poorly implemented post-discharge and there is often no TBIspecific outpatient follow-up, despite evidence that it is helpful. ${ }^{7,18,19}$ Furthermore, the lack of a formal TBI care pathway means that the majority of acute TBI patients not requiring critical or neurosurgical care are initially seen by clinicians who are not trained in the diagnosis and management of consequences at the level of pathology and neurological impairment. These patients, who present less dramatically, find themselves scattered throughout a hospital under various specialties (eg orthopaedics, general surgery, internal medicine or care of the elderly), and are subsequently discharged without specialist input or follow-up. This includes the rising numbers of elderly patients sustaining TBI through falls, whose injury severity may not even be initially recognised due to their altered physiology and the low energy mechanism of injury; TBI patients discharged directly from ED or presenting directly to community teams; and patients under acute medical and surgical specialties with polytrauma / other acute problems accompanied by unidentified TBI. ${ }^{20}$

TBI is often referred to as a 'hidden handicap' because disability after TBI results largely from cognitive, emotional and behavioural problems, rather than physical impairments. There is also the issue of the 'frontal lobe paradox' when patients perform well on routine screening but major problems emerge once in the community as a result of the 'saying-not-doing' / 'knowing-doing dissociation'. This is when patients know and say what they want and need to do, and yet fail to engage with therapy or perform necessary tasks, rapidly leading to, for example, loss of employment and relationship breakdown. ${ }^{21}$

All this frequently leads to inaccurate and delayed diagnoses, and poor understanding and recognition of the causes of disability after TBI, which often relate not only to the TBI but also to its interaction with underlying medical or psychological issues. Inevitably, subsequent rehabilitation and care is haphazard, management breaks down over time, the appearance of serious sequelae is not recognised nor investigated, and inappropriate or no treatment is initiated. This leads to preventable complications, slower recovery, lost opportunities for return to independence and productivity, and devastating personal and societal consequences. ${ }^{3-5}$ 
Fig 1. Disability after traumatic brain injury. ${ }^{7} \mathrm{~A}$ biopsychosocial model of the care needs of a patient after traumatic brain injury, reflecting a wide range of physical, cognitive emotional and behavioural issues. Based on The International Classification of Functioning, Disability and Health framework, created by the World Health Organization for measuring health and disability at individual and population levels. TBI $=$ traumatic brain injury.

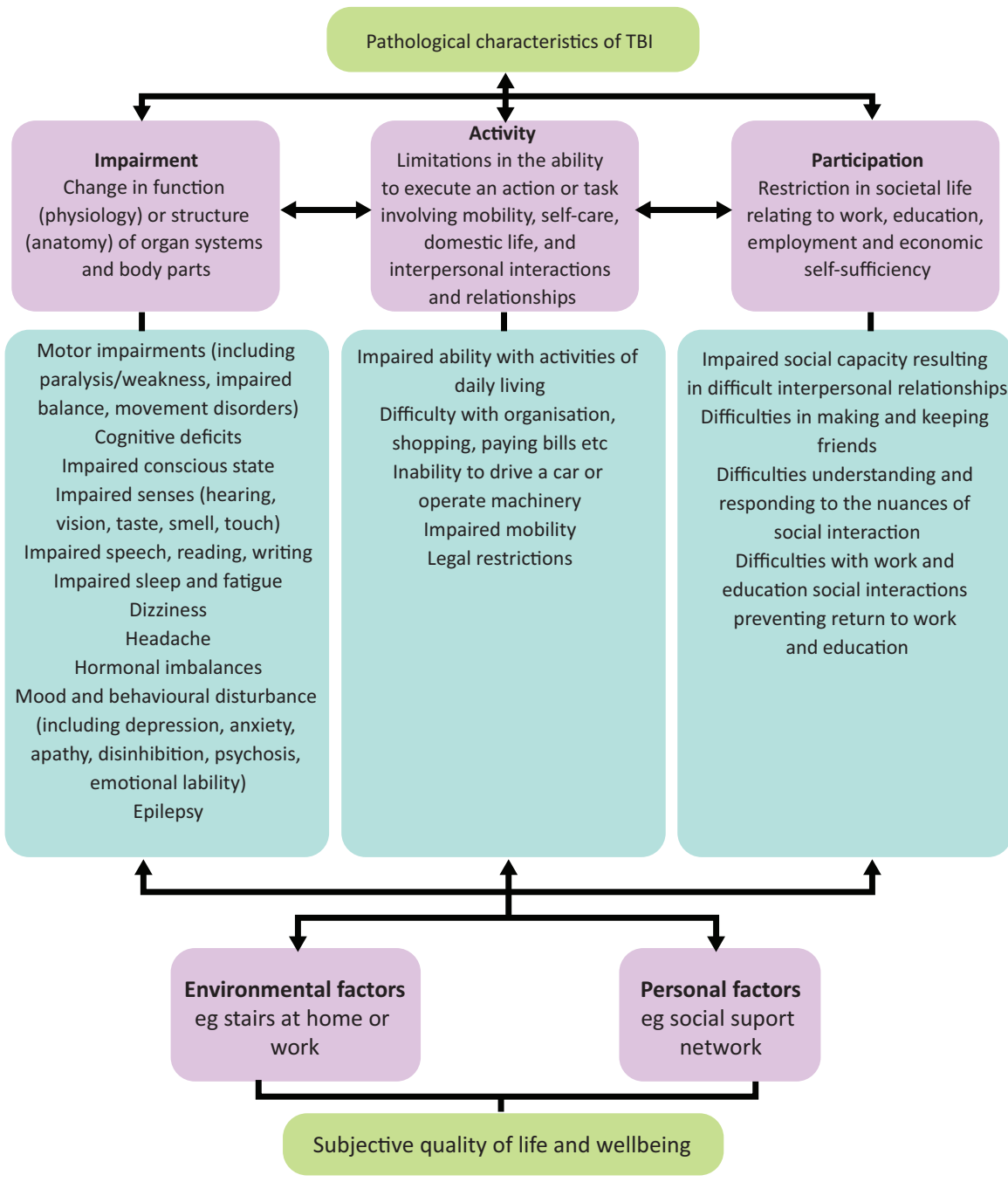

\section{Proposal}

Right diagnosis $\rightarrow$ targeted management $\rightarrow$ effective rehabilitation $\rightarrow$ better long-term life quality

Our aim is to improve practice within the current care pathway by reassigning responsibility for TBI patients of any severity to an 'expert leader'. ${ }^{22}$ This ensures that inpatients and outpatients become primarily the responsibility of a disorder-specific consultantled interdisciplinary team who are familiar with both the pathology of and the impairment-related diagnoses prevalent after TBI.

We propose the introduction of TBITs, led by a TBIC, within acute care in MTCs and trauma units (TU). TBITs will be involved

\section{Box 1. Levels of rehabilitation in the context of traumatic brain injury}

\section{Level 1}

Complex specialist rehabilitation services for patients with high physical dependency and/or mixed disability and/or cognitive behavioural issues. A TBI patient suitable for level 1 rehabilitation might have physical, cognitive and behavioural issues such as severe agitation, and often still be in post-traumatic amnesia.

\section{Level 2}

Specialist rehabilitation services for patients with less complex needs. A TBI patient suitable for level 2 rehabilitation might have ongoing cognitive or emotional or physical difficulties still requiring specialist rehabilitation or medical input but longer having significant behavioural issues (usually out of post-traumatic amnesia).

\section{Level 3}

Non- specialist rehabilitation services. A TBI patient suitable for level 3 rehabilitation might have ongoing re-enablement needs due to having TBI $+/-$ musculoskeletal injuries, but no longer require specialist medical input.

TBI = traumatic brain injury. 
early in care, initially jointly with surgical / neurosurgical / critical care teams, and will become the lead care team once immediate surgical or critical care needs are met. This will enable more consistent implementation of rehabilitation prescriptions and earlier coordinated discharge to level 1 rehabilitation units, and to rehabilitation networks developed in each of the $44 \mathrm{NHS}$ sustainability and transformation partnerships (STPs) or equivalent integrated care systems (ICS) where they exist. These networks should include access to level 2 and 3 specialist inpatient rehabilitation units delivering transitional and behavioural programmes, support to return to community settings, and a focus on promoting community independence, self-management, and early access to vocational rehabilitation. Neuro-navigation and case management should support and coordinate the pathway, and enable engagement with social care and community colleagues to provide specialist support workers and a training hub.

Seamless continuous care is likely to result in better patient outcomes and lower healthcare costs. For example, patients with strokes are admitted directly on to hyperacute stroke units (HASUs) under the care of clinicians specifically trained to have an understanding of stroke pathology and its consequences. The implementation of HASUs has been shown to improve both short- and long-term patient outcomes, performance standards and cost measures. ${ }^{23,24}$

A specific pathway for TBI will focus clinical attention, improve patient flow and outcomes, increase cohesion of the care pathway, reduce the cost of preventable disability and length of stay in acute beds, avoid disruption of the overall function of a trauma service, and release acute neurosurgical beds for patients with other pathologies. This pathway will also serve as a structure for data collection that can used to evaluate its clinical and economic impact, taking into account wider costs such as those of supporting services. The major trauma network has facilitated translational research, such as the studies of tranexamic acid in acute trauma. ${ }^{17}$ A TBI-specific pathway operating within the major trauma network will additionally encourage the formation of a knowledgebased network that enables translational research, trials and the implementation of new treatments at all stages of TBI.

\section{Clinical Pathway}

Our proposed clinical strategy for TBI underpins the development of this clinical pathway and services (Fig 2). We make the following recommendations.

> The clinical management of all patients who present after TBI of any severity, to any healthcare facility (hospital or community), is formulated early after injury by a neurosciencetrained clinician using individualised, impairment-based diagnostics and therapeutics. All TBI patients requiring inpatient care currently undergo pre-hospital triage by the emergency services. If necessary, admission will be to a regional MTC under the initial care of neurosurgical and critical care teams. After initial critical care and neurosurgical input, if required, acute patient management and care is the responsibility of an interdisciplinary TBIT, led by a TBIC. If the patient does not fulfil admission criteria to an MTC, and neurosurgical or critical care is not required or deemed unlikely, TBI cases may be admitted to a local TU for initial management by the ED team, in consultation with the MTC neurosurgeons and TBIT. Management of acute TBI patients who do not initially present to MTCs / neurosciences units will depend on patient condition and availability of neurological assessment and diagnostics. If the patient presents to a centre with limited inpatient neurology service and neurological diagnostics, or deteriorates unexpectedly, they may be immediately transferred to the MTC / neurosciences centre. In other cases, the patient may be first assessed by the on-call neurology team and then discussed with the MTC-based TBIC. The TBIT and TBIC will have a consulting role for non-MTC hospitals and trauma units, and patients should be discussed with them early. This will be via ad hoc phone consultations, and also expanded use of the tele-consults / online multidisciplinary team meetings (MDTs) promoted by the COVID-19 pandemic. For example, the TBIT at the MTC may host weekly MDTs to which patients at other hospitals can be referred and discussed. If the patient requires rapid access rehabilitation or early inpatient rehabilitation, they can be considered for transfer. We also propose that in non-MTC hospitals, there should be satellite TBITs, composed of small numbers of specialist nurses/ therapists who can help advise on care and facilitate access to all steps of the rehabilitation pathway.

> In the MTC, the TBIC/T will operate via a rapid access acute rehabilitation unit or acute brain injury unit (ABIU), into which patients would be admitted once they have no further critical care and surgical needs or, if appropriate, directly from ED. The TBIT will direct acute and early assessment and treatment, develop rehabilitation goals and plan onward referral to later rehabilitation via the rehabilitation prescription. Consulting support of other neuroscience, rehabilitation and trauma disciplines will be sought when required.

> The TBIC/T will ensure that appropriate referrals are initially outlined at discharge, and subsequently updated. Referrals will be documented in a patient-held rehabilitation prescription. These referrals will enable access, via neuro-navigators (Box 2) and case managers, to longer-term treatment, rehabilitation and care. ${ }^{25}$

> The TBIC/T will run specialist TBI outpatient clinics and a multidisciplinary outreach team, which will provide postdischarge follow-up and rehabilitation. The clinics will also see patients discharged from non-MTCs, and directly referred from ED, general practice and other community resources. The clinics would have consulting responsibilities within specialist community-based placements providing late rehabilitation and long-term placement after TBI.

> The TBIC/T, with the input of neuro-navigators and case managers, will develop and maintain links to relevant specialist services. These will include alcohol and drug rehabilitation services, domestic violence services, and relevant third-sector services (such as local charities). These services would provide a 'liaison' service to assist where necessary, and would include inperson reviews, contribution to MDTs and outpatient referrals. Where such services do not exist, members of the TBIC/T should seek to act as catalyst for the creation of such services.

The disability of TBI survivors is often masked by their good physical recovery. This pathway (Fig 2) provides a conditionspecific consultant-led multidisciplinary team able to accurately disentangle and diagnose and treat the wide range of problems presented by TBI. This disorder-specific model should, as has been seen with stroke, improve patient outcomes and optimise flow through both acute services and the wider network of rehabilitation units and community-based services. 


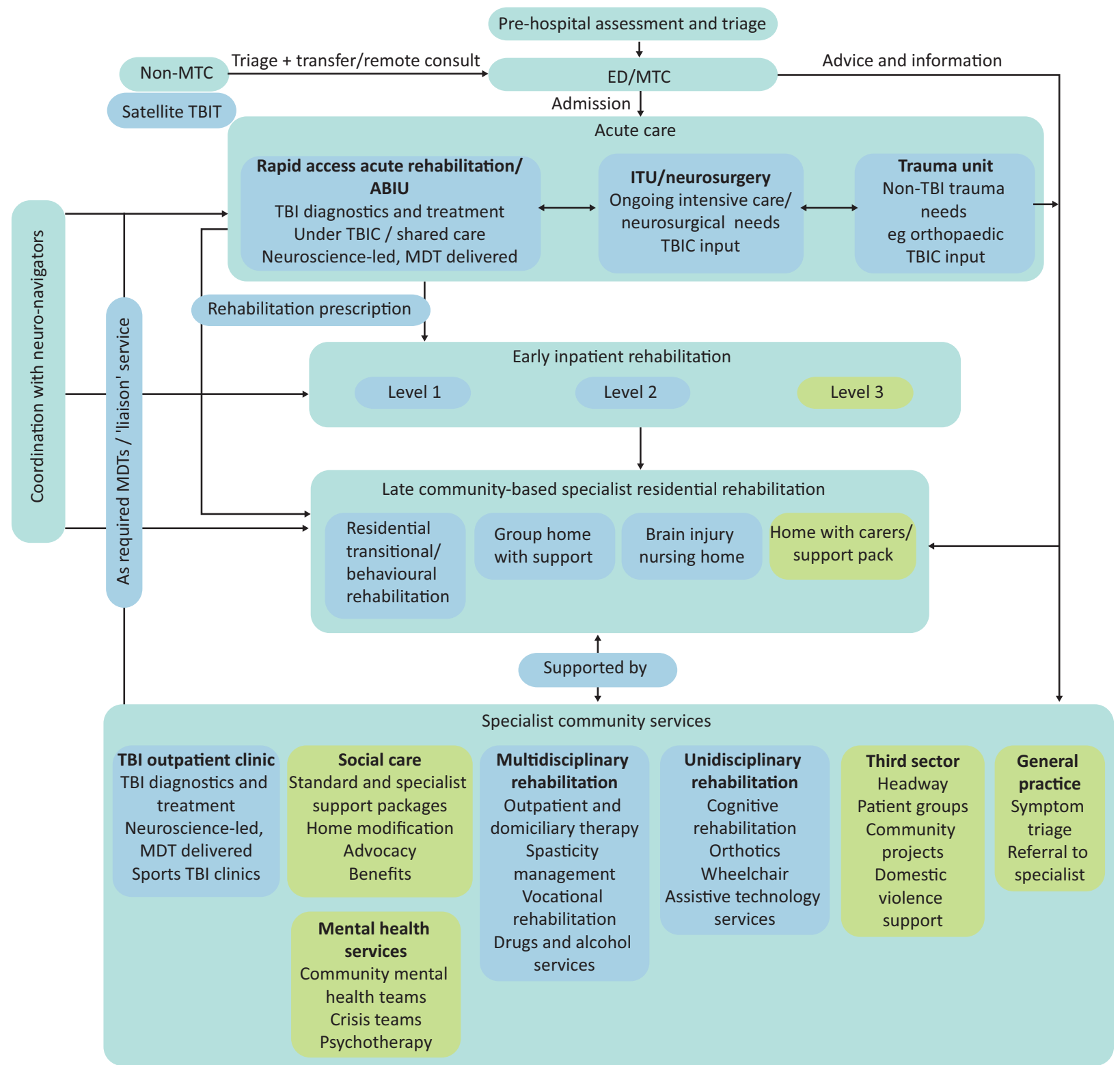

Fig 2. Proposed traumatic brain injury care pathway. Schematic of patient flow outlined in our proposal. Blue boxes indicate how the TBIC and TBIT would interact with and enhance the current systems. ABIU = acute brain injury unit; $E D=$ emergency department; ITU = intensive care unit; $M D T=$ multidisciplinary team meeting; MTC = major trauma centre; $\mathrm{TBI}=$ traumatic brain injury; $\mathrm{TBIC}=$ traumatic brain injury consultant; $\mathrm{TBIT}=$ traumatic brain injury team.

Implementation of this pathway for patients after TBI will enable:

> comprehensive neuroscience-based assessment and diagnosis at the level of pathology and impairment early after presentation

> appropriate longer-term prognoses and treatments based on the International Classification of Functioning, Disability and Health (ICF) framework (Fig 1)

$>$ timely and targeted goal setting during acute, early and late rehabilitation

$>$ continuity of care and treatment from hospital into the community

> longer-term support and treatment appropriate to their needs
> a structure for evaluation of TBI services, and also facilitate collaboration with research institutions.

The preferred model for funding this pathway would be via NHS England through the major trauma network, as this provides the most relevant 'hub' through which to organise and, if necessary, commission services. The commissioning of these services could be encouraged by making them part of the criteria to be awarded best practice tariff payments. Adequate specialist tariff funding must be made available to the MTCs in order to support this service, and the care at non-MTC sites. The TARN (www.tarn. ac.uk) database can serve as the basis for documenting TBI patients to enable specialist tariff funding as well as ongoing 
Box 2. What is a neuro-navigator? ${ }^{25}$

A neuro-navigator signposts patients and clinicians to appropriate services, with a focus on neurological conditions. Their aim is to get the patient to the right place, to access the right service, at the right time. Their responsibilities include:

> facilitating appropriate, smooth and timely transition for patient from hospital to community

> directing referrals to specialist local services (both inpatient and outpatient)

$>$ following patients over time to cater for changes in rehabilitation, medical or placement needs

$>$ advocating for patients as well as services.

The role is similar to that of an 'organisational case manager'. It focuses on clinical signposting, so requires a clinical background and organisational experience in a clinical setting, usually as a therapist, most often as an occupational therapist, but sometimes as a social worker or nurse.

audit and research. Other funding options include more ad hoc arrangements with local STPs/ICSs. However, this would require careful coordination and negotiation, and be at considerable risk of delay and competing interests.

\section{Staffing and training}

The TBIT will be multidisciplinary and led by a TBIC with specific neurosciences training. Neurosciences training is required to be able to accurately investigate and diagnose the many different possible contributory factors to disability after TBI. This is a prerequisite to directing appropriate management and referral to the right rehabilitation. While general rehabilitation training provides important skills, it does not provide adequate training in diagnosis of the most important and disabling complications of TBI (eg cognitive and neuropsychiatric presentations, and neurological problems such as complex seizure disorders, chronic headache and sleep disturbances). Physical disabilities, in which general rehabilitation provides the most training, are often a relatively minor problem after TBI. Therefore, simply increasing the number of general rehabilitation physicians is not the solution.

Instead, we anticipate that TBICs will come from a range of different 'feeder' specialties, including neurology, neurosurgery, psychiatry and rehabilitation. The approach should not be on a single specialty, but to recruit people with a number of key skills, who are willing to learn a wider skill set and undergo further training once in post, as required. Within the same unit, there would ideally be a mix of 'feeder' specialties so that TBICs can each contribute their expertise and learn from each other. The multidisciplinary team would also include occupational and physical therapists, neuropsychologists and clinical psychologists, social services, complex discharge coordinators, and clinical nurse specialists.

National roll out of such a programme would necessitate increasing the number of appropriately trained consultants. One such possibility is to provide a post-certificate of completion of training (CCT) fellowship in TBI, open to the whole range of 'feeder' specialties. Training would include TBI-specific rotations (eg through a neurocritical care unit at an MTC) as well as 'top-up' training in rehabilitation or psychiatry where it is not already part of the curriculum. There is already significant appetite for this within neurology trainees; one of the authors conducted a survey returned by 72 respondents which found that $75 \%$ agreed or strongly agreed with the sentence 'I would like to receive more specific training on managing acute and chronic TBI patients' and $32 \%$ 'probably would' or 'definitely would' apply for a post-CCT fellowship with rotations aimed at increasing experience in TBI (supplementary material S1). Additionally, $35 \%$ of respondents 'probably would' or 'definitely would' apply for a consultant job which involved TBI roles. For neurosurgery, TBI is also an appropriate subspecialty, and recruitment of neurosurgeons into TBIC roles would help reduce the number of CCT holders who do not have substantive posts ( 43 in 2018). ${ }^{26}$

Creation and publication of guidelines are often a catalyst to improving services and staffing. Taking the stroke HASU as a model, at its initial implementation, there were certainly not enough clinicians available for every proposed HASU, but sufficient in the major centres to provide 'proof of concept'. This has then led to recruitment elsewhere, and concomitant expansion of the supporting therapy services. For example, the British Association of Stroke Physicians (BASP) have been able to use the HASU pathway to demonstrate ongoing staff shortage and thus to call for further recruitment and, crucially, training. ${ }^{27}$ Leveraging the current major trauma networks to trial our proposal would help provide the data to support further rollout, along with training and recruitment.

\section{Health economics}

A streamlined, coordinated approach to TBI care is likely to result in economic savings from improved patient flow through hospitals and improved long-term outcomes. This is particularly important as TBI patients are often of working age. The implementation of HASUs for management of strokes has produced cost savings, for example, due to shorter hospital stays. ${ }^{23}$

Studies of service delivery after TBI are relatively sparse, but there is evidence to suggest that an approach such as the one we propose is likely to produce substantial cost savings and improve care efficiency. A retrospective study of 9,000 neurosurgical bed occupancy days found that the lack of organised acute TBI service with rehabilitation capability led to $72 \%$ of TBI patients remaining in neurosurgical beds when they no longer required neurosurgical management. ${ }^{28} \mathrm{~A}$ Norwegian cohort study compared outcomes in two groups of patients who had received acute rehabilitation and were transferred either directly to sub-acute rehabilitation or, as a result of bed availability, via alternative routes. ${ }^{29}$ This study found shorter hospital stays and better functional outcomes in those who had direct transfer to sub-acute rehabilitation. The experience at St George's Hospital acute traumatic brain injury pathway, which is a neuroscienceled service, has been that inpatient stay duration and costs for TBI patients are reduced (by $37 \%$ and $£ 30,000$, respectively) by timely rather than delayed transfer to an ABIU (Griffin C, Dilley M, personal communication, 2018).

Furthermore, studies have consistently demonstrated that any upfront investments made in early specialist rehabilitation services are rapidly offset by the cost savings made through increased and faster functional improvements. An Irish study found that costs of early inpatient rehabilitation were recovered on average within 30 months, with annual savings of $\sim € 20,000$ per patient. ${ }^{30}$ In the 
most dependent group, costs were recovered within 15.6 months, and thereafter savings of over $€ 50,000$ per patient accrued annually. Data from the UK Rehabilitation Outcome Collaborative shows that early after ABIU inpatient rehabilitation saved about $\leq 27,000$ per annum per patient and paid for itself over 19 months after discharge, while rehabilitation of medically unstable ('hyperacute') patients paid for itself after 28 months and saved about $£ 24,000$ per annum per patient. ${ }^{31,32}$ Late residential rehabilitation for patients with neurobehavioural disorders after TBI improves functional outcomes and is estimated to result in lifetime savings of up $£ 1.13$ million. ${ }^{33}$ A prospective randomised study found that a specialist multidisciplinary domiciliary outreach team showed increased independence and lessened care needs compared with usual care, suggesting that specialist communitybased rehabilitation late after TBI also results in significant longterm cost savings. ${ }^{34}$

\section{Summary}

We propose a pathology and impairment-based conditionspecific approach to the management of TBI, delivered by a multidisciplinary team, and led by a clinician with TBI-specific competencies and experience. This pathway will be centred around neurosciences and MTCs, but the TBIT will also provide ad hoc and formal consults for acute TBI patients presenting elsewhere, with transfer as required. This is to ensure that patients initially admitted to non-MTC sites can also access the same downstream rehabilitation and outpatient services. This approach will focus clinical attention, improve management of TBI patients as well as polytrauma patients with a TBI, improve patient flow by increasing cohesion and unblocking acute beds, and avoid disruption to the major trauma service. This will, in turn, improve clinical outcomes, enhance patient experience, and reduce overall costs associated with TBI. Encompassing all TBI care under a single team will also enable efficient collation of data for service evaluation and patient outcomes, which will provide a more effective means for evaluating interventions and translation of novel research findings.

\section{Supplementary material}

Additional supplementary material may be found in the online version of this article at www.rcpjournals.org/clinmedicine: S1 - Survey results and free-text comments.

\section{Acknowledgements}

Peter ] Hutchinson is supported by the National Institute for Health Research and Royal College of Surgeons of England.

\section{Conflicts of interest}

Paul Cooper is chair the Association of British Neurologists Traumatic Brain Injury Advisory Group.

\section{References}

1 National Institute for Health and Care Excellence. Head injury: assessment and early management: Clinical guideline [CG176]. NICE, 2014.

2 Skandsen T, Einarsen CE, Normann I et al. The epidemiology of mild traumatic brain injury: the Trondheim MTBI follow-up study. Scand J Trauma Resusc Emerg Med 2018;26:34.
3 Parsonage M. Traumatic brain injury and offending: an economic analysis. Centre for Mental Health, 2016. www.centreformentalhealth.org.uk/publications/traumatic-brain-injury-and-offending

4 Fazel S, Wolf A, Pillas D et al. Suicide, fatal injuries and other cause of premature mortality in patients with traumatic brain injury; a 41-year Swedish population study. JAMA Psychiatry 2014;71: 326-33.

5 Olesen J, Gustavsson A, Svensson M et al. The economic cost of brain disorders in Europe. Eur J Neurol 2012;19:155-62.

6 Sharp DJ, Scott G, Leech R. Network dysfunction after traumatic brain injury. Nat Rev Neurol 2014;10:156-66.

7 World Health Organization. How to use the ICF: A practical manual for using the International Classification of Functioning, Disability and Health (ICF). WHO, 2013.

8 Engberg AW, Teasdale TW. Psychosocial outcome following traumatic brain injury in adults: a long-term population-based followup. Brain Injury 2004;18:533-45.

9 Williams WH, Chitsabesan P, Fazel S et al. Traumatic brain injury: a potential cause of violent crime? Lancet Psychiatry 2018;5:836-44.

10 Dilley M, Avent C. Long-term neuropsychiatric disorders after traumatic brain injury. In: Uehara T (ed), Psychiatric disorders - worldwide advances. InTech, 2011.

11 Godbolt AK, Stenberg M, Jakobsson J et al. Subacute complications during recovery from severe traumatic brain injury: frequency and associations with outcome. BMJ Open 2015;5:e007208.

12 Polinder S, Cnossen MC, Real RGL et al. A multidimensional approach to post-concussion symptoms in mild traumatic brain injury. Front Neurol 2018;9:1113.

13 Kashluba S, Paniak C, Blake T et al. A longitudinal, controlled study of patient complaints following treated mild traumatic brain injury. Arch Clin Neuropsychol 2004;19:805-16.

14 Hou R, Moss-Morris R, Peveler R et al. When a minor head injury results in enduring symptoms: a prospective investigation of risk factors for postconcussional syndrome after mild traumatic brain injury. J Neurol Neurosurg Psychiatry 2012;83:217-23.

15 Maas AIR, Menon DK, Adelson PD et al. Traumatic brain injury: integrated approaches to improve prevention, clinical care, and research. Lancet Neurology 2017;16:987-1048.

16 Sampalis ]S, Denis R, Lavoie A. Trauma care regionalization: a process-outcome evaulation. J Trauma 1999;46:565-79.

17 Moran CG, Lecky F, Bouamra O et al. Changing the system - major trauma patients and their outcomes in the NHS (England) 200817. EClinicalMedicine 2018;2-3:13-21.

18 Singh R, Ventakeshwara G, Batterley ], Bruce S. Early rehabilitation in head injury: can we improve the outcomes? Arch Trauma Res 2013;2:103-7.

19 British Society of Rehabilitation Medicine. Specialised neurorehabilitation service standards. BSRM, 2015.

20 Coats T. Blunt major trauma: now two different diseases? Br J Hosp Med 2020;81:1-3.

21 Worthington A. Decision making and mental capacity: Resolving the frontal paradox. Neuropsychologist 2019;7:31-5.

22 Goodall A, Stoller JK. The future of clinical leadership: evidence for physician leadership and the educational pathway for new leaders. BMJ 2017:1:8-11.

23 Morris S, Hunter RM, Ramsay AIG et al. Impact of centralising acute stroke services in English metropolitan areas on mortality and length of hospital stay: Difference-in-differences analysis. BM] 2014; 349:94757.

24 Melnychuk M, Morris S, Black G et al. Variation in quality of acute stroke care by day and time of admission: Prospective cohort study of weekday and weekend centralised hyperacute stroke unit care and non-centralised services. BMJ Open 2019;9:e025366.

25 Brown S, Carolan J. The role of neuro-navigators in London. Improving the pathway after brain injury, a presentation at ABIL conference, 2016. www.abil.co.uk/wp-content/uploads/2016/11/ ABIL-Sept-2016-conference-presentations.pdf 
26 Sinha S, McKenna G, Whitfield P et al. Workforce planning in neurosurgery. Br J Neurosurg 2020:34:3-8.

27 British Association of Stroke Physicians. Meeting the future consultant workforce challenges: stroke medicine. BASP, 2019.

28 Bradley LJ, Kirker SGB, Corteen E et al. Inappropriate acute neurosurgical bed occupancy and short falls in rehabilitation: implications for the National Service Framework. $\mathrm{Br}$ ] Neurosurg 2006:20:36-9.

29 Andelic N, Bautz-Holter E, Ronning P et al. Does an early onset and continuous chain of rehabilitation improve the long-term functional outcome of patients with severe traumatic brain injury? J Neurotrauma 2012;29:66-74.

30 Cooney MT, Carroll A. Cost effectiveness of inpatient rehabilitation in patients with brain injury. Clin Med 2016;16:109-13.

31 Turner-Stokes L, Williams H, Bill A et al. Cost-efficiency of specialist inpatient rehabilitation for working-aged adults with complex neurological disabilities: a multicentre cohort analysis of a national clinical data set. BMJ Open 2016;6:e010238.
32 Turner-Stokes L, Bavikatte G, Williams H, et al. Cost-efficiency of specialist hyperacute in-patient rehabilitation services for medically unstable patients with complex rehabilitation needs: a prospective cohort analysis. BMJ Open 2016;6:e012112.

33 Oddy M, da Silva Ramos S. The clinical and cost-benefits of investing in neurobehavioural rehabilitation: a multi-centre study. Brain Injury 2013;27:1500-7.

34 Powell J, Heslin J, Greenwood R. Community based rehabilitation after severe traumatic brain injury: a randomised controlled trial. J Neurol Neurosurg Psychiatry 2002;72:193-202.

Address for correspondence: Dr Richard Greenwood, National Hospital for Neurology and Neurosurgery, Queen Square, London WC1N 3BG, UK.

Email: richard.greenwood2@nhs.net 